\title{
A RADON-NIKODYM THEOREM FOR NATURAL CONES ASSOCIATED WITH VON NEUMANN ALGEBRAS. II
}

\author{
HIDEKI KOSAKI ${ }^{1}$
}

\begin{abstract}
A natural cone associated with a ( $\sigma$-finite) von Neumann algebra is considered. Let $\xi_{0}$ be a cyclic and separating vector in the cone. For each vector $\xi$ in the cone, there always exists a positive selfadjoint operator $t$ affiliated with the algebra satisfying $\xi=\downarrow \downarrow W \xi_{0}$. Certain uniqueness results on $t$ for a given $\xi$ are also obtained.
\end{abstract}

1. Introduction. We consider a ( $\sigma$-finite) von Neumann algebra $\mathfrak{N}$ acting standardly on a Hilbert space $\mathcal{H}$. Let $\mathcal{P}^{\natural}$ be a natural cone in $\mathcal{K}$, and $J$ be the corresponding modular conjugation, that is, the quadruple $\left(\mathscr{R}, \mathcal{H}, J, \mathcal{P}^{\natural}\right)$ is a standard form, $[2,3,4]$. We fix a cyclic and separating vector $\xi_{0}$ in $\mathcal{P}^{\natural}$ so that $\mathcal{P}^{\natural}$ may be expressed as the closure of all vectors $x J x J \xi_{0}, x \in \mathfrak{R}$.

Improving the main result in [5], we will prove

THEOREM. For each vector $\xi$ in $\mathcal{P}^{\natural}$, there always exists a positive selfadjoint operator $t$ affiliated with $\mathfrak{T}$ satisfying $\xi_{0} \in \mathcal{O}(t), J t \xi_{0}=J t J \xi_{0} \in \mathscr{D}(t)$, and $\xi=t J t J \xi_{0}$.

In $\S \S 4$ and 5 , we will also prove that (i) the above $t$ is not uniquely determined by $\xi$ as an operator $(\$ 5)$, (ii) however, it is unique in a certain sense (Lemma 5, Proposition 6)

We will use certain terminologies and properties established in [5], which are summarized in the next section. Our standard references on the modular theory and unbounded operators are [9 and 7] respectively.

2. Preliminaries [5]. The quadruple $\left(\mathscr{T}, \mathcal{H}, J, \mathscr{P}^{\natural}\right)$ and a distinguished cyclic and separating vector $\xi_{0}$ in $\mathcal{P}^{\natural}$ with the vector functional $\phi_{0}=\omega_{\xi_{0}} \in \mathfrak{R}_{*}^{+}$will be fixed throughout.

For each $\chi \in \mathscr{T}_{*}^{+}$, its unique implementing vector in $\mathcal{P}^{\natural}$ is denoted by $\xi_{\chi}$. (Thus, $\chi=\omega_{\xi_{x}}$ and $\xi_{\phi_{0}}=\xi_{0}$.) The operator $x \xi_{0} \in \mathfrak{N} \xi_{0} \rightarrow J x^{*} \xi_{\chi} \in \mathfrak{T}^{\prime} \xi_{x}$ is positive and essentially selfadjoint, and its closure is denoted by $\Delta_{\chi \phi_{0}}^{1 / 2}$. When $\chi=\phi_{0}$, we write

$$
\Delta=\Delta_{\phi_{0}}=\Delta_{\phi_{0} \phi_{0}} \text {, the usual modular operator }[9] \text {. }
$$

Received by the editors April 13, 1982 and, in revised form, June 25, 1982.

1980 Mathematics Subject Classification. Primary 46L10.

'Supported in part by the National Science Foundation, grant number MCS-8102158. 
We denote the set of all entire elements in $\mathscr{R}$ (resp. $\mathfrak{R}^{\prime}$ ) for the modular automorphism group $\sigma_{t}=\operatorname{Ad} \Delta^{i t}$ (resp. $\sigma_{t}^{\prime}=\operatorname{Ad} \Delta^{-i t}$ ), $t \in \mathbf{R}$, simply by $\mathscr{R}_{0}$ (resp. $\left.\mathfrak{T}_{0}^{\prime}\right)$.

In what follows, we will also fix an (arbitrary) vector $\xi$ in $\mathcal{P P}^{\natural}$, and set $\phi=\omega_{\xi} \in$ $\Re_{*}^{+}$. Hence, we will sometimes write $\xi_{\phi}$ instead of $\xi$. In [5], we set

$$
\psi(x)=\left(\Delta^{1 / 4} x \xi_{0} \mid \xi_{\phi}\right), \quad x \in \Re,
$$

and proved $\psi \in \mathscr{R}_{*}^{+}$and

LemMa 1 ([5. Lemma 3.3]). The unique implementing vector $\xi_{\psi}$ for $\psi$ in $\mathscr{S}^{\natural}$ belongs to $\mathscr{D}\left(\Delta^{1 / 4}\right) \cap \mathcal{D}\left(\Delta^{-1 / 4}\right)$.

As usual, we denote the closure of the positive cone $\mathbb{R}_{+} \xi_{0}$ (resp. $\mathscr{N}_{+}^{\prime} \xi_{0}$ ) in $\mathscr{K}$ by $\mathscr{P}^{\sharp}=\mathscr{P}_{\phi_{0}}^{\sharp}\left(\right.$ resp. $\left.\mathcal{P}^{b}=\mathscr{P}_{\phi_{0}}^{b}\right)[9]$. It is easy to check

$$
\begin{gathered}
\mathcal{P}^{\sharp}=\left\{\zeta \in \mathcal{H} ;(\zeta \mid \eta) \geqslant 0 \text { for all } \eta \in \mathscr{P}^{b}\right\}=J^{\mathscr{P} b}, \\
J \zeta=\Delta^{1 / 2} \zeta(\text { resp. } J \zeta=\zeta) \text { if } \zeta \in \mathcal{P}^{\sharp}\left(\text { resp. } \zeta \in \mathcal{P P}^{\natural}\right), \\
\mathcal{P P}^{\sharp} \subseteq \Delta^{-1 / 4}\left(\mathcal{P P}^{\natural} \cap \mathscr{D D}\left(\Delta^{-1 / 4}\right)\right), \Delta^{1 / 4} \mathcal{P P}^{\sharp} \subseteq \mathcal{P P}^{\natural} .
\end{gathered}
$$

(See $[2,9]$ for details.)

We will frequently use the following result, which is an easy consequence of the spectral decomposition theorem:

LEMMA 2. Any core for a positive selfadjoint operator $h$ is also a core for $h^{\alpha}$, $0<\alpha<1$.

3. Proof of the Theorem. In this section, we will prove the Theorem stated in $\S 1$. after preparing two lemmas.

Based on Lemma 1, we set

$$
\zeta_{\phi}=\Delta^{-1 / 4} \xi_{\psi}
$$

This is a vector in ${ }^{\rho} \#$ (4). Let $a_{\phi}$ be a densely defined operator on $\mathcal{H}$ determined by

$$
\mathscr{D}\left(a_{\phi}\right)=\mathfrak{N}^{\prime} \xi_{0}, \quad a_{\phi}\left(x^{\prime} \xi_{0}\right)=x^{\prime} \xi_{\phi}, \quad x^{\prime} \in \mathfrak{N}^{\prime} .
$$

Obviously, $a_{\phi}$ is affiliated with $\mathfrak{T}$, and due to (2) it is a positive (symmetric) operator (so that it is closable).

Lemma 3. The vector $J \zeta_{\phi}\left(\in \mathcal{P P b}^{b},(2)\right)$ belongs to $\mathscr{D}\left(a_{\phi}^{*}\right)$, and $a_{\phi}^{*} J \zeta_{\phi}=\xi_{\phi}$.

Proof. It suffices to show that

$$
\left(J \xi_{\phi} \mid a_{\phi} x^{\prime} \xi_{0}\right)=\left(\xi_{\phi} \mid x^{\prime} \xi_{0}\right), \quad x^{\prime} \xi_{0} \in \mathscr{N}^{\prime} \xi_{0}=\mathscr{D}\left(a_{\phi}\right) .
$$

However, since $\mathfrak{R}_{0}^{\prime}$ is dense in $\mathfrak{R}^{\prime}$ with respect to the strong operator topology, it suffices to show the above equality for any $x^{\prime} \in \mathfrak{N}_{0}^{\prime}$. For such an $x^{\prime}$, we compute 


$$
\begin{aligned}
& \left(J \zeta_{\phi} \mid a_{\phi} x^{\prime} \xi_{0}\right)=\left(J \Delta^{-1 / 4} \xi_{\psi} \mid x^{\prime} \zeta_{\phi}\right)=\left(J \Delta^{-1 / 4} \xi_{\psi} \mid x^{\prime} \Delta^{-1 / 4} \xi_{\psi}\right) \\
& =\left(J \Delta^{-1 / 4} \xi_{\psi} \mid x^{\prime} \Delta^{-1 / 4} J \xi_{\psi}\right) \quad\left(\xi_{\psi} \in \mathrm{op}^{\natural} \text { and }(3)\right) \\
& =\left(J \Delta^{-1 / 4} \xi_{\psi} \mid x^{\prime} J \Delta^{1 / 4} \xi_{\psi}\right) \quad\left(J \Delta^{1 / 4} J=\Delta^{-1 / 4}\right) \\
& =\left(J x^{\prime} J \Delta^{1 / 4} \xi_{\psi} \mid \Delta^{-1 / 4} \xi_{\psi}\right) \\
& =\left(\sigma_{i / 4}\left(J x^{\prime} J\right) \xi_{\psi} \mid \xi_{\psi}\right) \\
& =\psi\left(\sigma_{i / 4}\left(J x^{\prime} J\right)\right) \\
& =\left(\Delta^{1 / 4} \sigma_{i / 4}\left(J x^{\prime} J\right) \xi_{0} \mid \xi_{\phi}\right) \\
& =\left(J x^{\prime} J \xi_{0} \mid \xi_{\phi}\right) \\
& =\left(\xi_{\phi} \mid x^{\prime} \xi_{0}\right) \quad((3)) . \quad \text { (Q.E.D.) }
\end{aligned}
$$

LEMmA 4. The vector $J \zeta_{\phi}$ belongs to the domain of the closure $\bar{a}_{\phi}$, and $\bar{a}_{\phi} J \zeta_{\phi}=\xi_{\phi}$.

Proof. The previous lemma and (3) yield

$$
\xi_{\phi}=J \xi_{\phi}=J a_{\phi}^{*} J \zeta_{\phi} .
$$

For each $n=1,2, \ldots$, let $x_{n}^{\prime}$ be $J a_{\phi}^{*} J e_{n}^{\prime}$, where $e_{n}^{\prime}$ is the spectral projection of $\left|J a_{\phi}^{*} J\right|=J\left(\bar{a}_{\phi} a_{\phi}^{*}\right)^{1 / 2} J$ corresponding to the interval $[0, n]$. Thus, $\left\{x_{n}^{\prime}\right\}_{n=1,2, \ldots}$ is a sequence in ' $M$ '.

Because of (5), one obtains

$$
\lim _{n \rightarrow \infty}\left\|x_{n}^{\prime} \zeta_{\phi}-\xi_{\phi}\right\|=\lim _{n \rightarrow \infty}\left\|x_{n}^{\prime} \zeta_{\phi}-J a_{\phi}^{*} J \zeta_{\phi}\right\|=0
$$

Also, since $J \xi_{0}=\xi_{0} \in \mathcal{D}\left(a_{\phi}\right)$ and $a_{\phi} \subseteq a_{\phi}^{*}$,

$$
J a_{\phi}^{*} J \xi_{0}=J a_{\phi} \xi_{0}=J \zeta_{\phi} .
$$

Thus, one obtains

$$
\lim _{n \rightarrow \infty}\left\|x_{n}^{\prime} \xi_{0}-J \zeta_{\phi}\right\|=\lim _{n \rightarrow \infty}\left\|x_{n}^{\prime} \xi_{0}-J a_{\phi}^{*} J \xi_{0}\right\|=0
$$

The above two convergences mean that the pair $\left(J \zeta_{\phi}, \xi_{\phi}\right)$ is in the closure of the graph $\Gamma\left(a_{\phi}\right)(\subseteq \mathcal{H} \oplus \mathcal{H})$. (Q.E.D.)

PROOF OF THE TheOREM. Since the closed operator $\bar{a}_{\phi}$ is positive, it admits a positive selfadjoint extension $t$ (see [1 or 8]). According to [8], $t$ is automatically affiliated with $\mathcal{O}$, since $a_{\phi}$ and $\bar{a}_{\phi}$ are. Because $a_{\phi} \subseteq \bar{a}_{\phi} \subseteq t$ and $J \zeta_{\phi}=J a_{\phi} \xi_{0}=J t \xi_{0}$, the Theorem follows from Lemma 4. (Q.E.D.)

4. Uniqueness. We will obtain certain uniqueness results on an operator $t$ appearing in the Theorem.

LeMma 5. $A$ vector $\zeta_{\phi}=t \xi_{0}\left(\in \mathcal{P P}^{\sharp}\right)$ is uniquely determined by a given $\xi=\xi_{\phi}$ in $\mathcal{P P}^{\natural}$ if $\xi_{\phi}=t J t J \xi_{0}$ as in the Theorem. 
Proof. When $\xi_{\phi}=t J t J \xi_{0}$ as in the Theorem, for each $x \in \mathscr{T}_{0}$, we compute

$$
\begin{aligned}
\psi(x) & =\left(\Delta^{1 / 4} x \xi_{0} \mid t J t J \xi_{0}\right) \quad(\text { recall }(1)) \\
& =\left(\sigma_{-i / 4}(x) \xi_{0} \mid t J t J \xi_{0}\right) \\
& =\left(\sigma_{-i / 4}(x) J t \xi_{0} \mid t \xi_{0}\right) \\
& =\left(x \Delta^{-1 / 4} J t \xi_{0} \mid \Delta^{1 / 4} t \xi_{0}\right) \\
& =\left(x \Delta^{1 / 4} t \xi_{0} \mid \Delta^{1 / 4} t \xi_{0}\right) \quad\left(t \xi_{0} \in \mathcal{P P}^{\sharp} \text { and }(3)\right) .
\end{aligned}
$$

Thus, $\Delta^{1 / 4} t \xi_{0} \in \operatorname{SP}^{\natural}((4))$ is the unique implementing vector $\xi_{\phi}$. Since $\Delta^{1 / 4}$ is nonsingular, the vector $\zeta_{\phi}=\Delta^{-1 / 4} \xi_{\psi}=t \xi_{0}$ is uniquely determined by $\xi_{\phi}$. (Q.E.D.)

PROPOSITION 6. Let $t$ be a positive selfadjoint operator affiliated with $\mathfrak{R}$ satisfying $\xi_{\phi}=t J t J \xi_{0} \in \mathcal{P P}^{\natural}$ (and $\xi_{0}, J t \xi_{0} \in \mathscr{D}(t)$ ). Then the (algebraic) product $\Delta^{1 / 4} t$ is densely defined and closable, and as an operator the closure $\left(\Delta^{1 / 4} t\right)^{-}$is uniquely determined by $\xi_{\phi}$. (Furthermore, $\left|\left(\Delta^{1 / 4} t\right)^{-}\right|$is exactly $\Delta_{\phi \phi_{0}}^{1 / 4}$. $)$

Proof. The first assertion is obvious due to

$$
\mathscr{N}_{0}^{\prime} \xi_{0} \subseteq \mathscr{D}\left(\Delta^{1 / 4} t\right), \quad \mathfrak{M}_{0}^{\prime} \xi_{0} \subseteq \mathscr{D}\left(t \Delta^{1 / 4}\right) \subseteq \mathscr{D}\left(\left(\Delta^{1 / 4} t\right)^{*}\right)
$$

For the second assertion, we at first note that

$$
\left(\Delta^{1 / 4} t\right)^{*}\left(\Delta^{1 / 4} t\right)^{-} \supseteq t \Delta^{1 / 4}\left(\Delta^{1 / 4} t\right)^{-} \supseteq t \Delta^{1 / 2} t .
$$

For each $x^{\prime} \in \mathfrak{N}_{0}^{\prime}$, we compute

$$
\begin{aligned}
t \Delta^{1 / 2} t x^{\prime} \xi_{0} & =t \Delta^{1 / 2} x^{\prime} t \xi_{0} \\
& =t \sigma_{i / 2}^{\prime}\left(x^{\prime}\right) J t \xi_{0} \quad\left(t \xi_{0} \in \mathcal{P}^{\sharp} \text { and }(3)\right) \\
& =\sigma_{i / 2}^{\prime}\left(x^{\prime}\right) t J t J \xi_{0} \\
& =\sigma_{i / 2}^{\prime}\left(x^{\prime}\right) \xi_{\phi}=J\left(J \sigma_{i / 2}^{\prime}\left(x^{\prime}\right) J\right) \xi_{\phi} \\
& =\Delta_{\phi \phi_{0}}^{1 / 2}\left(J \sigma_{i / 2}^{\prime}\left(x^{\prime}\right) J\right)^{*} \xi_{0}=\Delta_{\phi \phi_{0}}^{1 / 2} J \sigma_{-i / 2}^{\prime}\left(x^{*}\right) J \xi_{0} \\
& =\Delta_{\phi \phi_{0}}^{1 / 2} J \Delta^{-1 / 2} x^{\prime *} \xi_{0}=\Delta_{\phi \phi_{0}}^{1 / 2} x^{\prime} \xi_{0} .
\end{aligned}
$$

Here, we used $J \Delta^{-1 / 2} x^{\prime *} \xi_{0}=x^{\prime} \xi_{0}$ (see [9]). We thus proved that

$$
\left.\left(\Delta^{1 / 4} t\right)^{*}\left(\Delta^{1 / 4} t\right)^{-} \supseteq \Delta_{\phi \phi_{0}}^{1 / 2}\right|_{\pi_{0}^{\prime} \xi_{0}} .
$$

We recall that $\mathscr{N} \xi_{0}$ is a core for $\Delta_{\phi \phi_{0}}^{1 / 2}$. Based on the fact that $\mathfrak{R}_{0}$ is dense in $\mathscr{N}$ with respect to the strong*-operator topology, the definition of $\Delta_{\phi \phi_{0}}^{1 / 2}$ (in \$2) immediately implies that $\mathfrak{R}_{0} \xi_{0}\left(=\mathfrak{R}_{0}^{\prime} \xi_{0}\right)$ is also a core for $\Delta_{\phi \phi_{0}}^{1 / 2}$. Since $\left(\Delta^{1 / 4} t\right)^{*}\left(\Delta^{1 / 4} t\right)^{-}$is always (positive) selfadjoint, the above inclusion means that

$$
\left(\Delta^{1 / 4}\right)^{*}\left(\Delta^{1 / 4} t\right)^{-}=\left|\left(\Delta^{1 / 4} t\right)^{-}\right|^{2}=\Delta_{\phi \phi_{0}}^{1 / 2} .
$$

Thus, $\left|\left(\Delta^{1 / 4} t\right)^{-}\right|=\Delta_{\phi \phi_{0}}^{1 / 4}$ is uniquely determined by $\xi_{\phi}$. 
Because of Lemma 2, $\Delta_{\phi \phi_{0}}^{1 / 4}$ is essentially selfadjoint on $\mathfrak{T}_{0}^{\prime} \xi_{0}$. Thus the phase part $v$ of the polar decomposition $\left(\Delta^{1 / 4} t\right)^{-}=v\left|\left(\Delta^{1 / 4} t\right)^{-}\right|=v \Delta_{\phi \phi_{0}}^{1 / 4}$ is determined by

$$
\left\{\begin{array}{l}
v=0 \quad \text { on } \Re\left(\Delta_{\phi \phi_{0}}^{1 / 4}\right)^{\perp} \quad\left(=\left(\Re^{\prime} \xi_{\phi}\right)^{\perp}\right), \\
v\left(\Delta_{\phi \phi_{0}}^{1 / 4} x^{\prime} \xi_{0}\right)=\left(\Delta^{1 / 4} t\right)^{-} x^{\prime} \xi_{0}, \quad x^{\prime} \in \Re^{\prime} .
\end{array}\right.
$$

But, the right-hand side of the second equation is just $\Delta^{1 / 4} x^{\prime} t \xi_{0}$ due to the fact that $t$ is affiliated with the algebra. Lemma 5 thus implies that the phase part $v$ is also uniquely determined by $\xi_{\phi}$. (Q.E.D.)

Lemma 5 does not guarantee that $t$ itself is uniquely determined by $\xi_{\phi}$ as an operator (unless $\mathfrak{T}$ is finite, $[6,8]$ ). We will show this fact in the next section. We are thus convinced that Proposition 6 is a "correct" uniqueness property.

5. A counterexample. As stated in the last part of $\S 4$, we will show

Proposition 7. An operator $t$ in the Theorem is not uniquely determined by a given $\xi$ in $\mathcal{P P}^{\natural}$.

In fact, by constructing a counterexample, we will show that $t$ is not unique even when $\mathscr{R}$ is a factor of type $I_{\infty}$. Our example is an improved version of the one given in [6].

Let $\mathscr{K}$ be a separable Hilbert space, and $\mathscr{R}=B(\mathscr{K})$ acts on the Hilbert space $\mathcal{K}=C_{2}(\mathscr{K})$ (of all Hilbert-Schmidt class operators on $\mathscr{K}$ ) as left multiplications. Then, ( $\left.\mathscr{T}, \mathcal{K}, J={ }^{*}, \mathscr{P}^{\natural}=C_{2}^{+}(\mathscr{K})\right)$ is a standard form. In what follows, we will identify $C_{2}(\mathscr{K})$ with $\mathscr{K} \otimes \overline{\mathcal{K}}$ in the usual way. (For $\xi, \zeta \in \mathscr{K}, \xi \otimes \zeta$ is a rank one operator on $\mathcal{K}$ defined by $(\xi \otimes \zeta) \eta=(\eta \mid \zeta)_{\mathscr{K}} \xi, \eta \in \mathcal{K}$.) Thus, $\mathscr{N}$ may be considered as the tensor product $B(\mathscr{K}) \bar{\otimes} 1_{\mathscr{K}}$.

We choose and fix a closed symmetric positive operator $t$ on $\mathscr{K}$ satisfying $(t \xi \mid \xi)_{\mathscr{K}} \geqslant\|\xi\|_{\mathscr{K}}^{2}, \xi \in \mathscr{D}(t)$, with two different positive selfadjoint extensions $t_{1}$ and $t_{2}$. (In fact, suppose that $b$ is a positive closed operator which is not essentially selfadjoint. Then, the Friedrich extension $(b+1)_{\infty}$ and the Krein extension $(b+1)_{0}$ of $b+1$ are always different, [1].) We also fix a dense sequence $\left\{e_{n}\right\}_{n=1.2 \ldots}$ in $\mathscr{D}(t)$. Thanks to the well-known Schmidt orthogonalization process, we may and do assume that $\left\{e_{n}\right\}(\subseteq \mathscr{D}(t))$ is an orthonormal basis for $\mathcal{K}$. We then choose a sequence $\left\{\lambda_{n}\right\}_{n=1,2, \ldots}$ of strictly positive numbers satisfying

$$
\begin{gathered}
\lambda_{n} \leqslant 1 / n, \\
\lambda_{n}\left\|t e_{n}\right\|_{\Re} \leqslant 1 / n^{2}, \\
\lambda_{n}\left\|t e_{n}\right\|_{\Re}^{2} \leqslant 1 / n^{2} .
\end{gathered}
$$

At first, (6) means that $\xi_{0}=\sum_{n=1}^{\infty} \lambda_{n} e_{n} \otimes e_{n}$ gives rise to an element in $\mathcal{H}=$ $C_{2}(\mathcal{K})=\mathscr{K} \otimes \overline{\mathcal{K}}$. This is a nonsingular positive Hilbert-Schmidt class operator on $\mathscr{K}$ so that $\xi_{0} \in \mathcal{P P}^{\natural}$ is cyclic and separating. We now consider the closed positive operator $\tilde{t}=t \otimes 1$ affiliated with $\mathscr{R}=B(\mathcal{K}) \bar{\otimes}_{\mathfrak{K}}$ (see §VIII-10, [7], for tensor 
products of unbounded operators). The vector $\xi_{0}$ belongs to $\mathscr{D}(\tilde{t})$, and

$$
\tilde{t} \xi_{0}=\sum_{n=1}^{\infty} \lambda_{n}\left(t e_{n}\right) \otimes e_{n} \text {. }
$$

Indeed, the right-hand side is certainly a vector in $\mathcal{H}$ due to (7). Furthermore, (6) (resp. (7)) shows that $\sum_{n=1}^{m} \lambda_{n} e_{n} \otimes e_{n}\left(\right.$ resp. $\left.\tilde{t}\left(\sum_{n=1}^{m} \lambda_{n} e_{n} \otimes e_{n}\right)=\sum_{n=1}^{m} \lambda_{n}\left(t e_{n}\right) \otimes e_{n}\right)$ tends to $\xi_{0}$ (resp. $\left.\sum_{n=1}^{\infty} \lambda_{n}\left(t e_{n}\right) \otimes e_{n}\right)$ as $m$ goes to $\infty$. Since $J(\xi \otimes \zeta)=(\xi \otimes \zeta)^{*}=\zeta$ $\otimes \xi, \xi, \zeta \in \mathcal{K}$, we have

$$
J \tilde{t} \xi_{0}=\sum_{n=1}^{\infty} \lambda_{n} e_{n} \otimes\left(t e_{n}\right) .
$$

Repeating the above arguments together with (7) and (8), we then have

$$
J \tilde{t} \xi_{0} \in \mathscr{D}(\tilde{t}), \quad \tilde{t} \tilde{t} \xi_{0}=\sum_{n=1}^{\infty} \lambda_{n}\left(t e_{n}\right) \otimes\left(t e_{n}\right) \in \mathcal{P P}^{\natural} .
$$

Thus, two different positive selfadjoint operators $\tilde{t}_{1}=t_{1} \otimes 1$ and $\tilde{t}_{2}=t_{2} \otimes 1$ affiliated with $\Re$ enjoy

$$
\tilde{t}_{1} J \tilde{t}_{1} \xi_{0}=\tilde{t}_{2} J \tilde{t}_{2} \xi_{0} \quad\left(=\sum_{n=1}^{\infty} \lambda_{n}\left(t e_{n}\right) \otimes\left(t e_{n}\right) \in \operatorname{\rho pp}^{n}\right) .
$$

ACKnowledgement. The author wishes to thank Professor Huzihiro Araki for useful comments which simplified some arguments in the original version of the present article.

\section{REFERENCES}

1. A. Alonso and B. Simon, The Birman-Krein-Visik theory of self-adjoint extensions of semi-bounded operators. J. Operator Theory 4 (1980), 251-270.

2. H. Araki, Some properties of modular conjugation operators of von Neumann algebras and a non-commutative Radon-Nikodym theorem with a chain rule, Pacific J. Math. 50 (1974), 309-354.

3. A. Connes, Caracterisation des espaces vectoriels ordonnés sous-jacents aux algèbres de von Neumann. Ann. Inst. Fourier (Grenoble) 24 (1974), 121-155.

4. U. Haagerup, The standard form of von Neumann algebras, Math. Scand. 37 (1975), 271-283

5. H. Kosaki, A Radon-Nikodym theorem for natural cones associated with von Neumann algebras. Proc. Amer. Math. Soc. 84 (1982), 207-211.

6. F. Perdrizet, Elements positifs relatifs à une algèbre hilbertienne à gauche, Compositio Math. 23 (1971), 25-47.

7. M. Reed and B. Simon, Methods of modern mathematical physics. I, Academic Press, New York, San Francisco and London, 1972.

8. C. Skau, Positive self-adjoint extensions of operators affiliated with a von Neumann algebra, Math. Scand. 44 (1979), 175-195.

9. M. Takesaki, Tomita's theon of modular Hilhert algebras and its applications, Lecture Notes in Math., vol. 128, Springer-Verlag, Berlin, Heidelberg and New York, 1970.

Department of Mathematics, University of Kansas, Lawrence, Kansas 66045

Current address: Department of Mathematics, Purdue University, West Lafayette, Indiana 47907 